\title{
Stationary object detection in forward scattering radar
}

\begin{abstract}
This paper proposes ground object detection based on Forward Scattering Radar (FSR) system. The main discussion is the effect having transmitter move in a circular motion to scan area of interest. Most of the published papers on practical FSR were based on the fixed transmitter. By having rotating transmitter, the signals received contain extra information carried by the Doppler due to relative movement of the transmitter-taget-receviver. The flexibility of transmitter to move increases the coverage of FS networks and suitable to detect fixed object or slow moving object. The paper analysed this concept by detecting fixed object on the ground and it is suitable for debris detection on the airport runway.
\end{abstract}

Keyword: Forward scattering radar; Object detection 